\title{
SAFETY, EFFICACY AND FEASIBILITY OF TOTAL TUBELESS PERCUTANEOUS NEPHROLITHOTOMY FOR RENAL AND UPPER URETERIC CALCULUS
}

\author{
Bhalaguru Iyyan Arumugam¹, Puvai Murugan P2, Anandan Murugesan', Tracy Rosalin Poulose ${ }^{4}$ \\ ${ }^{1}$ Assistant Professor, Department of Urology, PSG Institute of Medical Sciences and Research. \\ ${ }^{2}$ Assistant Professor, Department of Urology, PSG Institute of Medical Sciences and Research. \\ 3 Professor, Department of Urology, PSG Institute of Medical Sciences and Research. \\ 4Junior Resident, Department of Surgery, PSG Institute of Medical Sciences and Research.
}

\begin{abstract}
BACKGROUND

Percutaneous Nephrolithotomy (PCNL) is the gold standard in the treatment of renal and upper ureteric calculus, but involves the placement of nephrostomy tube and Double J stent to aid in drainage of the kidney. The placement of Nephrostomy tube and Double J stent has their own drawbacks like post-operative discomfort, prolonged hospitalisation, pain and stent related symptoms. Hence, we evaluated the safety, efficacy and feasibility of Total Tubeless Percutaneous Nephrolithotomy (TTPCNL) in uncomplicated PCNL patients.
\end{abstract}

\section{MATERIALS AND METHODS}

Patient undergoing PCNL for renal and upper ureteric calculus were recruited for the study. Preoperatively, patients with congenital anomalies, previous surgery, coagulopathy and staghorn renal calculi were excluded. At the end of the PCNL procedure, the surgeon decided not to place nephrostomy tube and Double J stent in patients with minimal bleeding and no residual calculus as determined by nephroscopy and fluoroscopy. Procedure was considered as success in the absence of residual calculus or if the residual calculus size was less than $4 \mathrm{~mm}$. Complications were graded according to Clavien-Dindo classification.

\section{RESULTS}

210 TTPCNL was performed in our Institute from March 2010 to July 2016. The mean stone size was 17 mm (Range $10 \mathrm{~mm}-35$ $\mathrm{mm}$ ). Mean operative time was 52 mins. (35 mins. - 90 mins.), 5 patients had 2 access tracts for complete stone clearance and mean hospital stay was 2 days ( 1 - 4 days). The success rate in stone clearance was $91 \%$, while 12\%, 4\%, 6\% had Grade 1, 2, 3 ClavienDindo complication respectively and no Grade 4 or 5 Clavien-Dindo complication.

\section{CONCLUSION}

TTPCNL is a safe, effective and feasible technique for selective renal and upper ureteric stones. Double J stents and nephrostomy tracts are reserved for the specific situation.

\section{KEYWORDS}

Percutaneous Nephrolithotomy, Renal Calculus, Minimally Invasive Surgery.

HOW TO CITE THIS ARTICLE: Arumugam BI, Murugan PP, Murugesan A, et al. Safety, efficacy and feasibility of total tubeless percutaneous nephrolithotomy for renal and upper ureteric calculus. J. Evolution Med. Dent. Sci. 2016;5(91):6786-6789, D0I: $10.14260 /$ jemds/2016/1533

\section{BACKGROUND}

Percutaneous Nephrolithotomy (PCNL) is the gold standard treatment for renal calculi. In 1976, Fernstrom and Johansson performed the first removal of a renal stone percutaneously. ${ }^{1}$ Standard PCNL includes percutaneous access to the pelvicalyceal system with fragmentation of the stone and retrieval of the stone fragments with placement of nephrostomy tube and Double J stent. Post-operatively, nephrostomy tube placement reduces the primary haemorrhage and urinary extravasation. Placement of

Financial or Other, Competing Interest: None.

Submission 27-09-2016, Peer Review 01-11-2016,

Acceptance 07-11-2016, Published 14-11-2016.

Corresponding Author:

Dr. Puvai Murugan P,

Assistant Professor,

Department of Urology,

PSG Institute of Medical Sciences and

Research, Avinashi Road,

Peelamedu, Coimbatore-641004.

E-mail: puovai@gmail.com

DOI: $10.14260 /$ jemds $/ 2016 / 1533$ nephrostomy tube also acts as an access for second procedure in the presence of residual calculi. But placement of nephrostomy tube and Double J stents in patients undergoing PCNL causes post-operative discomfort, prolonged hospitalisation, pain and stent related symptoms. Tubeless PCNL means placement of Double J stent without nephrostomy tube. Total tubeless PCNL is defined as PCNL without nephrostomy tube and Double J stent. The aim of our study is to evaluate the safety, effectiveness and feasibility of total tubeless percutaneous nephrolithotomy.

\section{MATERIALS AND METHODS}

Between March 2010 and July 2016, 809 patients underwent PCNL for renal and proximal ureteric calculi; among these only 210 patients had total tubeless PCNL. Patients with radioopaque calculi in the kidney and proximal ureter were included in our study. Patients with congenital anomalies, previous renal surgery, coagulopathy and staghorn renal calculi were excluded in our study. All patients were evaluated with clinical history, serum creatinine, blood sugar, blood urea, serum calcium, serum uric acid, serum electrolytes and coagulation profile. Radiological investigations like ultrasound 
Kidney, Ureter and Bladder (KUB), x-ray KUB or non-contrast Computed Tomography KUB were done. PCNL was performed in prone position under general anaesthesia. Before prone positioning, cystoscopy was done to deploy 5-Fr ureteric catheter into the renal system. Contrast media was injected into the ureteric catheter to identify the pelvicalyceal anatomy. Percutaneous puncture was made under fluoroscopic guidance of the desired calyx using 18G needle. Once the needle was confirmed in the desired calyx, 0.035 thermo guide wire was inserted into the pelvicalyceal system or some time down to the bladder under fluoroscopy. Central rod was placed along the thermo guide into the pelvicalyceal system to steady the guide wire. Alken serial dilators were used to perform dilatation and 28-Fr Amplatz sheath was introduced into the collecting systems (Figure 1). Using 24-Fr rigid nephroscope, pelvicalyceal system was inspected and stone fragmented with pneumatic lithoclast and fragments was removed. Clearance was checked under fluoroscopy and nephroscopy. A decision was made to insert Double J stent and/or Nephrostomy tube based on surgeons' assessment of bleeding and presence of residual calculi. Track infiltration was done using $0.25 \%$ of Bupivacaine with 23 -Gauge needle at 6-o'clock and 12-o'clock position for post-operative analgesia. Amplatz sheath was removed at the end of the procedure. Site of the track was sutured with 1-0 silk suture.

Non-steroidal anti-inflammatory drug (NSAID's - Injection Diclofenac $75 \mathrm{mg}$ deep IM) was used as first line analgesic therapy. Narcotic drugs (Injection Fortwin 30 micrograms) were used as second line analgesia. Postoperative complication such as fever, flank pain, haematuria, hydrothorax and analgesics requirements were recorded. Ultrasound KUB was done for all patients postoperatively on post-operative Day 1 (POD \#1) to look for residual calculi, hydroureteronephrosis and perinephric collection. Foley catheter was removed on POD \#1. Followup imaging to assess the success of PCNL was done at one week postoperatively.

\section{RESULTS}

210 patients ( 164 male and 46 female) with mean age of 42 years and mean stone size of $17 \mathrm{~mm}$ underwent total tubeless PCNL, of which15 patients had serum creatinine more than 1.5 $\mathrm{mg} / \mathrm{dL}$. Stone was located in renal pelvis $(\mathrm{n}=54)$, upper calyx $(\mathrm{n}=14)$, middle calyx $(\mathrm{n}=46)$, lower calyx $(\mathrm{n}=64)$ and proximal ureter $(n=37)$. Major number of patients had inferior calyceal puncture $(n=148)$. Five patients had two puncture tracks; 38 patients had multiple renal calculi. Mean operative duration was 52 minutes (35 - 75 minutes). The demographic profiles of the patients are shown in Table 1.

Two patients had simultaneous total tubeless PCNL. Five patients had 2 access tracts (Figure 2); mean hospital stay was 2 days (Range 1 - 6 days). Immediate stone free rate was 91\%. The average residual stone burden was $5.5 \mathrm{~mm}$ (Range $4-7$ $\mathrm{mm}$ ). Mean return to normal activity took 9.8 days (6 - 21 days). The Clavien-Dindo classification for surgical complications is given in Table 2; $4 \%$ of the patients had DJ stenting secondary to persistent fever and hydroureteronephrosis on USG KUB; $2 \%$ of the patients had extracorporeal shockwave lithotripsy and flexible ureteroscopy with laser lithotripsy for residual calculi.

\begin{tabular}{|c|c|}
\hline Mean (or) N & TTPCNL \\
\hline Total Patients & 210 \\
\hline Laterality (U:B) & 208:02 \\
\hline Age, Yrs. & 42 \\
\hline Sex - Male:Female & $164: 46$ \\
\hline Stone Size $\mathrm{mm}$ & 17 \\
\hline \multicolumn{2}{|l|}{ Serum Creatinine mg/dL } \\
\hline$<1.5$ & 195 \\
\hline$>1.5$ & 15 \\
\hline \multicolumn{2}{|l|}{ Access } \\
\hline Upper Calyceal Puncture & 14 \\
\hline Middle Calyceal Puncture & 53 \\
\hline Inferior Calyceal Puncture & 148 \\
\hline \multicolumn{2}{|l|}{ No. of Tracts } \\
\hline One & 205 \\
\hline Two & 5 \\
\hline \multicolumn{2}{|l|}{ Outcome (Mean) } \\
\hline Operative Duration (Min) & $52(35-75)$ \\
\hline Analgesia mg - Diclofenac & $75(0-200)$ \\
\hline Hospital Stay (Days) & 2 \\
\hline \multicolumn{2}{|l|}{ Side } \\
\hline Right & 106 \\
\hline Left & 109 \\
\hline \multicolumn{2}{|l|}{ Size of Amplatz (N) } \\
\hline $30 \mathrm{Fr}$ & 32 \\
\hline $28 \mathrm{Fr}$ & 154 \\
\hline $26 \mathrm{Fr}$ & 26 \\
\hline \multicolumn{2}{|l|}{ Outcome \% } \\
\hline Stone Free Status (\%) & $91 \%$ \\
\hline $\mathrm{N}=$ With Fragmentation & 168 \\
\hline $\mathrm{N}=$ Without Fragmentation & 47 \\
\hline Retreatment (\%) & $2 \%$ \\
\hline Residual Fragments $<5 \mathrm{~mm}$ & $3 \%$ \\
\hline Return to Normal Activity (Days) & 9.8 \\
\hline
\end{tabular}

\begin{tabular}{|c|c|c|}
\hline Grade & Definition & \\
\hline $\begin{array}{l}\text { Grade } \\
\quad 1\end{array}$ & $\begin{array}{l}\text { Any deviation from the normal course } \\
\text { without the need for pharmacological } \\
\text { treatment or surgical, endoscopical and } \\
\text { radiological intervention allowed therapeutic } \\
\text { regimens are: drugs as anti-emetic, } \\
\text { electrolytes and physiotherapy }\end{array}$ & $12 \%$ \\
\hline $\begin{array}{l}\text { Grade } \\
\quad 2\end{array}$ & $\begin{array}{l}\text { Requiring pharmacological treatment with } \\
\text { drugs other than such allowed for Grade } 1 \\
\text { complication. Blood transfusions and } \\
\text { total parenteral nutrition are also included }\end{array}$ & $4 \%$ \\
\hline $\begin{array}{c}\text { Grade } \\
3 \\
\end{array}$ & $\begin{array}{c}\text { Requiring surgical, endoscopic } \\
\text { or radiological intervention }\end{array}$ & $6 \%$ \\
\hline $3 a$ & Intervention not under general anaesthesia & $4 \%$ \\
\hline $3 \mathrm{~b}$ & Intervention under general anaesthesia & $2 \%$ \\
\hline $\begin{array}{l}\text { Grade } \\
\quad 4\end{array}$ & $\begin{array}{c}\text { Life-threatening complications (Including } \\
\text { CNS complications) requiring } \\
\text { ICU management }\end{array}$ & nil \\
\hline $4 \mathrm{a}$ & Single organ dysfunction (Including dialysis) & nil \\
\hline $4 \mathrm{~b}$ & Multiorgan dysfunction & nil \\
\hline $\begin{array}{c}\text { Grade } \\
5\end{array}$ & Death of a patient & Nil \\
\hline & $\begin{array}{l}\text { Table 2. The Clavien-Dindo Classification } \\
\text { for Surgical Complications }\end{array}$ & \\
\hline
\end{tabular}




\begin{tabular}{|c|c|}
\hline Advantages & Disadvantages \\
\hline Decrease postoperative pain & $\begin{array}{c}\text { Ureteric obstruction - } \\
\text { clots/residual calculi }\end{array}$ \\
\hline Less analgesia dosage & $\begin{array}{c}\text { Need for new access in } \\
\text { significant residual calculi }\end{array}$ \\
\hline Short hospital stay & $\begin{array}{c}\text { Loss of tamponade in } \\
\text { significant bleeding }\end{array}$ \\
\hline $\begin{array}{c}\text { Minimal urinary leakage - } \\
\text { Fast wound healing }\end{array}$ \\
\hline No stent related symptoms \\
\hline $\begin{array}{c}\text { Avoid cystoscopy to } \\
\text { remove the Double J stent }\end{array}$ \\
\hline \multicolumn{2}{|c|}{ Table 3. Advantages and Disadvantages } \\
of Total Tubeless PCNL
\end{tabular}

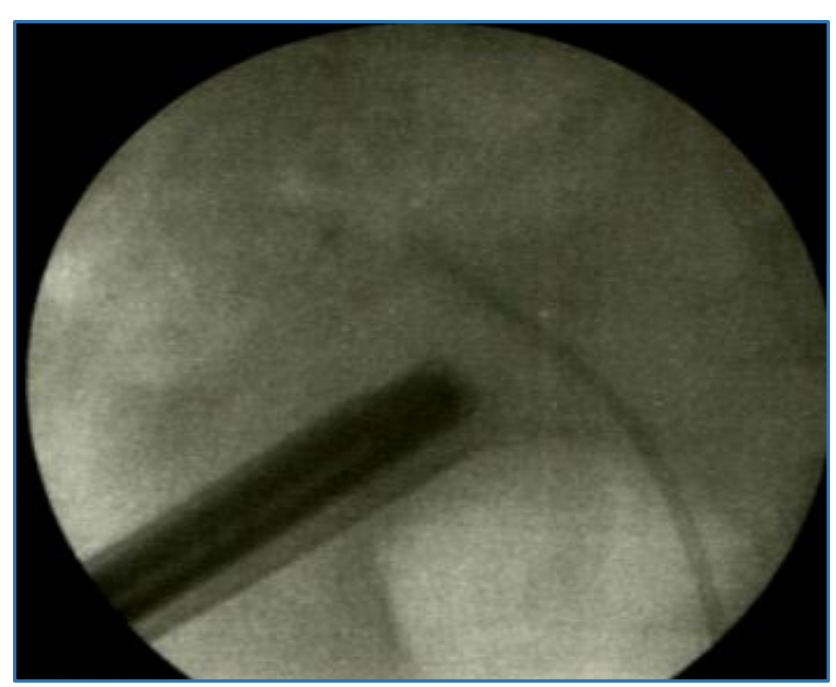

Figure 1. Percutaneous Access of Pelvicalyceal System

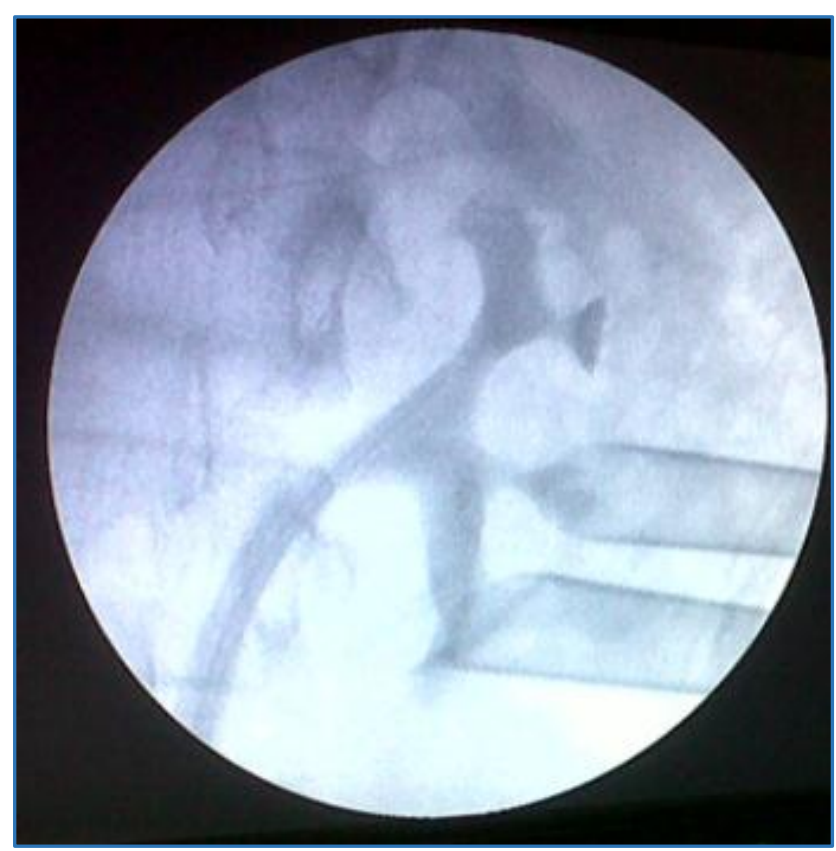

Figure 2. Double Access

\section{DISCUSSION}

Bellman et $\mathrm{al}^{2}$ first reported tubeless PCNL in 112 patients and compared with standard PCNL later concluded that tubeless PCNL has less post-operative discomfort, short hospitalisation and speedy recovery. The treatment cost of tubeless procedure was low when compared to the standard procedure. Advantages and disadvantages of total tubeless PCNL are outlined in Table 3.

Aghamies et $\mathrm{al}^{3}$ concluded that tubeless PCNL is feasible and more advantageous than standard PCNL for uncomplicated renal calculi. The indications of total tubeless PCNL were,

1. Single access tract.

2. Minimal blood loss.

3. No perforation in pelvicalyceal system.

4. No second look nephroscopy.

5. No residual calculi under fluoroscopy and nephroscopy.

Sung II Yun et al ${ }^{4}$ compared between Standard and Totally Tubeless Percutaneous Nephrolithotomy found that totally tubeless PCNL group showed a shorter hospitalisation and a lesser analgesic requirement compared with the standard PCNL group. Blood loss and change in creatinine were not significantly different between these two groups. Crook et $\mathrm{al}^{5}$ randomly performed standard PCNL and totally tubeless PCNL on 50 patients with renal stones and reported that there were no significant intergroup differences in haemorrhage, infection, blood transfusion or clinical values, but that the hospitalisation time was shorter in the totally tubeless PCNL group than in the standard PCNL group.

Hasan et $\mathrm{al}^{6}$ reported that there were no major operative or postoperative complications in both TTPCNL group and standard PCNL group. But postoperative fever was detected in 2 patients in standard PCNL group and managed medically using antibiotic prophylaxis and no urosepsis was developed. No patients had urinoma or perirenal haematoma. Nadali et al ${ }^{7}$ reported two patients of total tubeless PCNL had high-grade fever, for which DJ stenting was done. In our study, five patients had two access tracts. Eight patients had DJ stenting post-operatively in view of acute pyelonephritis.

Gupta et $\mathrm{al}^{8}$ found that all 96 patients had stone free rate with no complication. Aghamies et al $^{3}$ in 43 patients of total tubeless PCNL, no patient had any complication and residual calculi. In our study six patients had residual calculus, four patients had Extracorporeal Shockwave Lithotripsy (ESWL) and two patients had Retrograde Intra-Renal Surgery (RIRS) and at third month post-operatively all the patients had complete stone clearance. Totally tubeless PCNL reduced the additional cost and discomfort associated with removal of the stent. Limitation of the tubeless procedure is that it precludes secondary procedures for the treatment of residual stones.

Guanglu Song et $\mathrm{al}^{9}$ showed that recovery time was significantly shorter for patients receiving tubeless minipercutaneous nephrolithotomy than those treated with standard PCNL in pre-school children with renal calculi. Treating preschool children with tubeless percutaneous nephrolithotomy has advantages over standard PCNL including faster recovery and shorter hospital stay.

Guohua Zeng et $\mathrm{l}^{10}$ reported that it is a safe and effective treatment modality for children with large upper urinary tract stone disease and there was no significant difference in the surgical outcome and complications as compared to adult population. The author noted that the stone-free rate in paediatric patients had good results as in adults without an increase of complication rate. He advised that multiple percutaneous nephrostomy tracts should be practiced with caution in children with upper urinary tract stone disease. 
Sabnis et al ${ }^{11}$ advocated 'Mini-Microperc' which is a further technical modification, in which an 8-Fr sheath is used to allow insertion of ultrasonic or pneumatic lithoclast probe with suction. It is safe and efficient in the management of small renal calculi in adult and paediatric population. Ureteric catheter was removed along with Foley catheter in first postoperative day. Patient was discharged in first post-operative day if there was no complication.

\section{CONCLUSION}

Total tubeless PCNL has favourable outcome in selected patients if stone burden is less than $3 \mathrm{~cm}$, no significant residual stones, no significant pelvicalyceal perforation and minimal bleeding. Total tubeless PCNL has advantages of decreased postoperative pain, less analgesia requirement, short hospital stay and no requirement for a secondary procedure like cystoscopy for stent removal.

\section{REFERENCES}

1. Patel SR, Nakada SY. The modern history and evolution of percutaneous nephrolithotomy. J Endourol 2015;29(2): 153-7.

2. Bellman GC, Davidoff R, Candela J, et al. Tubeless percutaneous renal surgery. J Urol 1997;157(5):1578-82.

3. Aghamir SM, Hosseini SR, Gooran SJ. Totally tubeless percutaneous nephrolithotomy. J Endourol 2004;18(7): 647-8.
4. Yun SI, Lee YH, Kim JS, et al. Comparative study between standard and totally tubeless percutaneous nephrolithotomy. Korean J Urol 2012;53(11):785-9.

5. Crook TJ, Lockyer CR, Keoghane SR, et al. A randomized controlled trial of nephrostomy placement versus tubeless percutaneous nephrolithotomy. J Urol 2008;180(2):612-4.

6. Goktug HN, Yesil S, Ozturk U, et al. Totally tubeless percutaneous nephrolithotomy- selecting for success in children. Adv Clin Exp Med 2013;22(4):565-70.

7. Moosanejad N, Isapour A, Fazli M, et al. Effectiveness of totally tubeless percutaneous nephrolithotomy: a retrospective study. International J Med Invest 2014;3(4):123-6.

8. Gupta V, Sadasukhi TC, Sharma KK, et al. Tubeless and stentless percutaneous nephrolithotomy. BJU Int 2005;95(6):905-6.

9. Song G, Guo X, Niu G, et al. Advantages of tubeless minipercutaneous nephrolithotomy in the treatment of preschool children under 3 years old. J Pediatr Surg 2015;50(4):655-8.

10. Zeng G, Zhao Z, Wan S, et al. Comparison of children versus adults undergoing mini-percutaneous nephrolithotomy: large-scale analysis of a single institution. PLoS One 2013;8(6):e66850.

11. Sabnis RB, Ganesamoni R, Ganpule AP, et al. Current role of microperc in the management of small renal calculi. Indian J Urol 2013;29(3):214-8. 